\title{
Le logis en pan-de-bois dans les villes du bassin de la Meuse moyenne (1450 - 1650)
}

Liège, Commission royale des Monuments, Sites et Fouilles, dossier de la CRMSF $n^{\circ} 12,2008,314$ pages.

Joëlle Petit

\section{CpenEdition}

\section{Journals}

Édition électronique

URL : http://journals.openedition.org/dht/267

DOI : $10.4000 /$ dht.267

ISSN : 1775-4194

Éditeur :

Centre d'histoire des techniques et de l'environnement du Cnam (CDHTE-Cnam), Société des élèves du CDHTE-Cnam

Édition imprimée

Date de publication : 1 décembre 2009

Pagination : 226-228

ISBN : 978-2-9530779-4-0

ISSN : 0417-8726

\section{Référence électronique}

Joëlle Petit, «Le logis en pan-de-bois dans les villes du bassin de la Meuse moyenne (1450 - 1650)», Documents pour l'histoire des techniques [En ligne], $18 \mid 2^{\mathrm{e}}$ semestre 2009, mis en ligne le 24 septembre 2010, consulté le 22 septembre 2020. URL : http://journals.openedition.org/dht/267 ; DOI : https:// doi.org/10.4000/dht.267

Ce document a été généré automatiquement le 22 septembre 2020.

(c) Tous droits réservés 
Le logis en pan-de-bois dans les villes du bassin de la Meuse moyenne (1450 1650)

Liège, Commission royale des Monuments, Sites et Fouilles, dossier de la CRMSF $n^{\circ} 12,2008,314$ pages.

Joëlle Petit

\section{RÉFÉRENCE}

Le logis en pan-de-bois dans les villes du bassin de la Meuse moyenne (1450 - 1650), Liège, Commission royale des Monuments, Sites et Fouilles, dossier de la CRMSF $n^{\circ} 12,2008$, 314 pages. 
La Commission royale des Monuments, Sites et Fouilles de Belgique consacre ce dossier à un sujet encore peu étudié, les logis en pan-de-bois. La publication se fonde sur la thèse de doctorat de David Houbrechts soutenue en 2005 (Université de Liège, Histoire de l'art et archéologie). Pour disposer d'un corpus significatif, les techniques de charpenterie sont analysées à travers l'étude détaillée du logis urbain en pan-de-bois dans le bassin de la Meuse moyenne, du milieu du $\mathrm{XV}^{\mathrm{e}}$ au milieu du $\mathrm{XVII}^{\mathrm{e}}$ siècle, période marquée par les guerres et les destructions.

2 L'architecture en pan-de-bois est réinsérée dans le contexte socioéconomique de l'urbanisme médiéval et post-médiéval, notamment en lien avec l'exploitation et le commerce du bois (économie de gestion et d'environnement, transports et coûts de la mise en œuvre). Elle est également replacée dans le cadre de l'évolution de l'architecture civile afin de préciser les caractéristiques, la spécificité et l'évolution de ce patrimoine.

Ce travail constitue une première approche de la question; il tend à pallier le manque de références bibliographiques pour le patrimoine urbain, la maison paysanne étant examinée dans la collection Architecture rurale de Wallonie dirigée par Luc Francis Génicot ${ }^{1}$. Les sciences historiques ayant manifesté peu d'intérêt jusqu'à présent pour le secteur de la construction dans le bassin de la Meuse moyenne, les corpus de sources sont encore très fragmentaires.

4 L'approche de l'architecture en pan-de-bois relève essentiellement de l'archéologie du bâti, prolongeant les méthodes d'analyse des recherches effectuées sur les charpentes de toiture ${ }^{2}$. L'ouvrage comporte quatre parties, débutant chacune par un résumé ; il se clôture par un bilan de la méthode proposée, mise en perspective.

5 La première partie concerne la matière première : exploitation de la forêt, transport du bois (moyens de transport, circulation du bois sur la Meuse, marchés au bois), ainsi que façonnage des bois. Ce chapitre permet d'appréhender l'ensemble des opérations de débitage, d'équarrissage, de sciage, de marquage des bois, de façonnage et d'assemblage de la charpente, ainsi que le transport des pans-de-bois et le remontage in situ, à une époque où les bâtiments en pierre et en brique sont l'exception. L'auteur relève notamment les arbres sélectionnés pour leur forme et dimensions (courbure), le travail et l'organisation sur chantier, la description de l'outillage, le caractère préfabriqué des fenestrages, la technique de fabrication des pans-de-bois et les marques utilisées (flottage ou de marchands, équarissage, établissage, assemblage, montage des pans-debois, placement du clayonnage qui recevra le torchis et éclisses).

6 La deuxième partie de l'ouvrage examine les formes du logis en pan-de-bois. Elle est scindée en deux chapitres, respectivement relatifs à la période qui va des origines au bas Moyen-Age, puis à celle courant de 1450 à 1650. Les pans-de-bois primitifs étant peu 
nombreux et partiellement conservés, leur aspect pour les historiens reste vague. Quant aux matériaux utilisés pour les élévations en région mosane, ils restent inconnus, aucune élévation en pan-de-bois antérieure au XIV ${ }^{e}$ siècle n'ayant été identifiée.

7 Pour la période allant de 1450 à 1650, l'auteur examine d'abord les proportions, les plans et les volumes : disposition générale des bâtiments (morphologie, implantation et répartition), maisons dont la toiture est parallèle à la rue (formes principales), maisons sur pignon. Il étudie ensuite les toitures (toit, charpentes, couverture et protection des murs), les élévations : façades et pignons, suivant leur structure principale (usage des bois longs et des bois courts, structure principale, encorbellements, solins), leur structure secondaire (assemblages, ossatures) et l'éclairage naturel (baies, systèmes de fermeture, lucarnes), notamment identifié par le nombre de jours ouverts sur deux ou trois registres. L'auteur poursuit par l'identification de la décoration extérieure : bois (caractéristiques ornementales des ossatures fondées sur la répartition et le nombre de croix de Saint-André, qui semblent disparaître à la première moitié du XVII siècle), décors sculptés (planches de rives, pièces de charpente apparentes, consoles à décors sculptés pour le soutien des corniches). L'auteur termine par l'étude des autres types d'éléments décoratifs : épis de faîtage en plomb (métal, couleurs, formes) et par l'examen de l'espace intérieur (mal connu, étant donné les multiples transformations au cours des siècles) : circulation et escaliers, division, caves, cheminées, planchers et plafonds, décoration.

8 Cette deuxième partie expose les différentes techniques des pans-de-bois, datées par la dendrochronologie : les formes primitives par construction sur pilotis et à poteaux de fond; les constructions utilisant des fermes, plus évoluées, ainsi que les variantes, utilisation de fermes ou de sablières reposant directement sur le sol ou poteaux plantés dans le sol. Les observations sont essentiellement effectuées dans les vestiges d'immeubles, la structure originale étant en majorité constituée de murs pignons mitoyens, de planchers, de charpentes, difficiles à repérer car souvent recouverts de matériaux décoratifs et isolants. Les relevés des logis urbains déterminent qu'ils sont presque toujours composés de pans-de-bois sur les quatre faces, avec des systèmes de surplomb qui posent question sur la fonction de l'encorbellement et induisent des recherches sur les solins. Le système d'évacuation des eaux est en effet mal connu jusqu'à l'apparition des corniches, les toitures débordant de l'aplomb des murs. L'évolution des ossatures des pignons et des murs-de-refend avant le XVII ${ }^{\mathrm{e}}$ siècle est limitée à celle des ossatures de façade.

9 La troisième partie de l'ouvrage se rapporte à la vie des pans-de-bois : règlements, évolution des mentalités, phénomène de "pétrification", manque de reconnaissance. La quatrième partie est relative à l'évolution et à la spécificité du pan-de-bois mosan et au pan-de-bois dans son contexte. La reconstruction rapide de l'habitat à Dinant et Liège après les sacs du Moyen Âge pose question sur l'origine des savoir-faire, qui pourraient avoir été amenés par l'assaillant bourguignon. La Franche-Comté et la Bourgogne sont en effet les exemples les plus proches du bassin de la Meuse moyenne (décharges en croix de Saint-André, parenté des ossatures de façade).

10 L'auteur termine son ouvrage par un bilan quant à la méthode utilisée, mise en perspective. L'intérêt de l'étude du bâti en pan-de-bois par ces différents biais privilégie une approche matérielle notamment grâce à l'analyse archéologique, qui permet d'appréhender le bâtiment dans ses transformations successives et à la 
dendrochronologie, qui permet de situer les informations dans le temps. Le croisement des approches chronologique et dendrochronologique, replacé dans son contexte historique, économique et politique, met en évidence l'originalité des bâtiments en pan-de-bois du bassin de la Meuse moyenne, même si les recherches restent incomplètes. Le souhait de l'auteur est évidemment la poursuite de l'étude dans les régions limitrophes, afin de souligner les particularismes régionaux et la poursuite des recherches historiques aux archives et sur chantier.

11 Le dossier est complété et actualisé par quelques articles relatifs à des recherches récentes effectuées à Liège (Caroline Bolle, Jean-Marc Léotard, Guillaume Mora-Dieu), Dinant (Olivier Berckmans), Andenne (Jean-Louis Javaux), Manhay (Denis Henrotay) et Maastricht (Birget Dukers).

12 La documentation est importante par sa diversité et sa qualité. L'iconographie, très abondante, compte 379 illustrations (gravures, photos anciennes et récentes, détails de vestiges en place, plans, maquettes, dessins et relevés, modélisations et reconstitutions par infographie analytique des systèmes), outre celles relatives aux recherches récentes. L'ouvrage comporte 14 pages de bibliographie, un glossaire mentionnant les sources bibliographiques utilisées et un index des noms de lieux.

\section{NOTES}

1. Luc Francis Genicot, Patricia Butil, Sabine de Jonghe, Bernadette Lozet, Philippe Weber éd., Le patrimoine rural de Wallonie, La maison paysanne 1, des modèles aux réalités, Bruxelles, Namur, Commission royale des Monuments, Sites et Fouilles, 1986.

2. Patrick Hoffsummer, Les charpentes $d u X^{e}$ au XIXe siècle, typologie et évolution en France du Nord et en Belgique, Paris, Cahiers du Patrimoine $\mathrm{n}^{\circ}$ 62, 2002 et Les charpentes de toiture en Wallonie, typologie et dendrochronologie (XI $-\mathrm{XIX}^{e}$ siècle), Namur, Commission royale des Monuments, Sites et Fouilles, coll. Études et documents, monuments et sites 1, 1995.

\section{AUTEUR}

JOËLLE PETIT

CDHTE-Cnam 